\title{
64. Cellular Basis of Immunity. IV
}

\author{
Cross Immunity of the Mouse Mononuclear Phagocytes Immunized \\ with Live Vaccine of Salmonella choleraesuis and S. \\ typhimurium against the Infection with S. enteritidis \\ By Tokumitsu TanaKa, Ichiei Sato, Kazuko SaIto, \\ and Susumu Mitsuhashi \\ Department of Microbiology, School of Medicine, Gunma University \\ (Comm. by T. FuruhatA, M.J.A., June, 12, 1962)
}

In the preceding paper ${ }^{1)}$ cross immunity of the mouse mononuclear phagocytes immunized with live vaccine of Salmonella enteritidis was described.

Mononuclear phagocytes obtained from the mouse immunized with live vaccine of $S$. enteritidis, inhibited intracellular growth of S. ryphimurium, S. choleraesuis, S. enteritidis, and E. coli 0-55, regardless of the presence of immune sera. But the cells of normal mice as well as immunized mice with live or dead vaccine of $S$. enteritidis did not inhibit the intracellular multiplication of $M$. tuberculosis $H 37 R v$.

This paper deals with the cross immunity of mononuclear phagocytes of the mouse immunized with live vaccine of $S$. typhimurium or $S$. choleraesuis against the infection with $S$. enteritidis.

Materials and Methods. Experimental animals, immunization method, and antigenic structure of bacterial strains used were described in previous papers. ${ }^{1-4)}$ The tissue-culture medium and phagocytic system were reported in the preceding report. ${ }^{3)}$

Results. Cross-immunity of the mouse super-immunized with live vaccine of $S$. choleraesuis or $S$. typhimurium against the infection with S. enteritidis. As shown in Table I, mouse superimmunized with live vaccine of $S$. choleraesuis, S. enteritidis or S. typhimurium resisted completely the intravenous infection of 10 MLD virulent strain of the same organism used for immunization. But the mouse super-immunized with live vaccine of S. choleraesuis or S. typhimurium showed cross-immunity toward intravenous infection with the virulent strain of $S$. enteritidis.

Cellular resistance of mononuclear phagocytes derived from the mouse super-immunized with live vaccine. Mononuclear phagocytes derived from the mouse super-immunized with live vaccine of $S$. choleraesuis or S. typhimurium showed cross resistance against the infection of $S$. enteritidis. The results are shown in Fig. 1.

The normal mononuclear phagocytes were destroyed within 3 days 
of incubation by intracellular multiplication of the virulent strain 116-54 of S. enteritidis. Number of mononuclear phagocytes which were derived from normal or immunized mouse were calculated after

Table I. Cross immunity of the mouse super-immunized with live vaccine

\begin{tabular}{|c|c|c|c|}
\hline $\begin{array}{l}\text { Organism used for } \\
\text { immunization* }\end{array}$ & $\begin{array}{l}\text { Organism used for } \\
\text { challenge }\end{array}$ & $\begin{array}{l}\text { Number of } \\
\text { mice }\end{array}$ & $\underset{(\%)}{\text { Survival rate }}$ \\
\hline S. choleraesuis & S. enteritidis & 20 & 85 \\
\hline S. choleraesuis & S. choleraesuis & 10 & 100 \\
\hline S. enteritidis & S. choleraesuis & 20 & 75 \\
\hline S. enteritidis & S. enteritidis & 10 & 100 \\
\hline S. typhimurium & S. enteritidis & 10 & 70 \\
\hline S. typhimurium & S. typhimurium & 10 & 100 \\
\hline \multirow{3}{*}{$\begin{array}{l}\text { Non-immunized } \\
\text { control }\end{array}$} & S. enteritidis & 10 & 0 \\
\hline & S. choleraesuis & 10 & 0 \\
\hline & S. typhimurium & 10 & 0 \\
\hline
\end{tabular}

* S. enteritidis: Mice were super-immunized intravenously with $10^{-5} \mathrm{mg}$ of attenuated strain and $10^{-7} \mathrm{mg}$ of virulent strain of $S$. enteritidis at 21 days interval.

S. choleraesuis and S. typhimurium: Mice were super-immunized intravenously with $10^{-2} \mathrm{mg}$ of attenuated strain and $10^{-7} \mathrm{mg}$ of virulent strain of both strains at 21 days interval.

** Mice were challenged intravenously with $10^{-7} \mathrm{mg}$ of virulent strain.

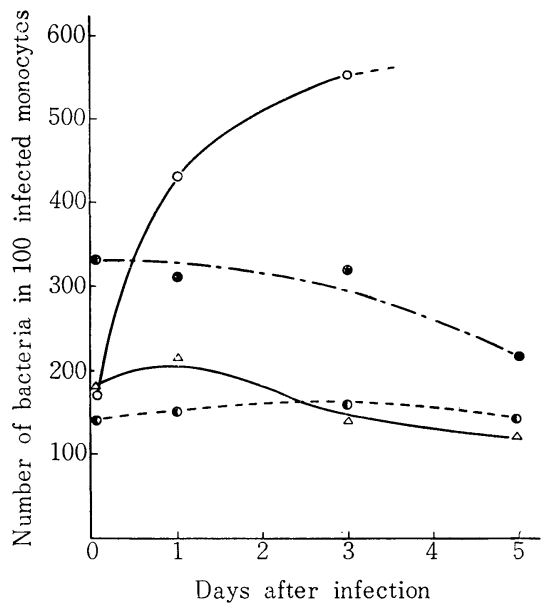

Fig. 1. Intracellular multiplication of the virulent strain 116-54 of $S$. enteritidis in normal monocytes and cross resistance of immune monocytes.

$\bigcirc-\bigcirc$, cells derived from normal mice; $--\cdots$, cells derived from mice super-immunized with live vaccine of $S$. choleraesuis; $1----1$, cells derived from mice super-immunized with live vaccine of $S$. typhimurium; $\triangle \longrightarrow \triangle$, cells derived from mice super-immunized with live vaccine of $S$. enteritidis. 
in vitro infection with the virulent strain 116-54 of S. enteritidis.

Monocytes derived from the mouse super-immunized with live vaccine of $S$. choleraesuis or $S$. typhimurium showed cellular resistance and did not show any decrease in cell number after infection, whereas normal monocytes showed about 80 per cent degeneration after 5 days of incubation. The results are shown in Fig. 2.

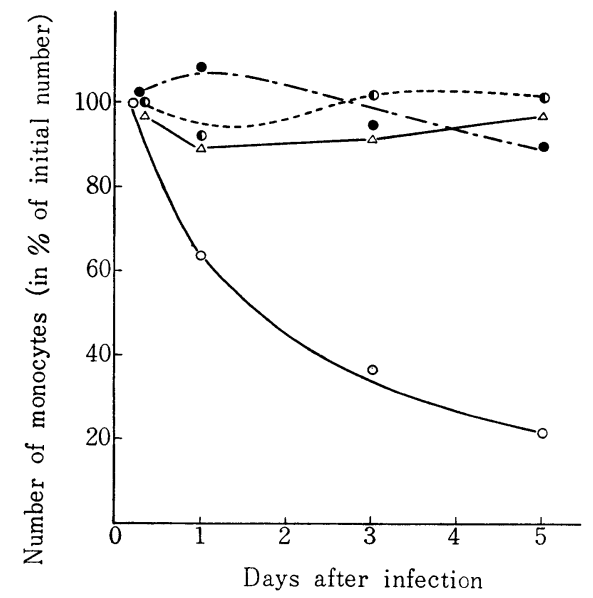

Fig. 2. Number of monocytes after infection with the virulent strain 116-54 of S. enteritidis, which were derived from normal or immunized mouse.

See foot-note of Fig. 1. The number of monocytes is expressed in percent of initial number. The total number of monocytes (ca. 500) in 5 microscopic fields $(20 \times 7 \times 1.5)$ were calculated in each experiment. Each point indicates the mean of three experiments.

Discussion. The degeneration of normal mononuclear phagocytes caused by infection with $M$. tuberculosis $H 37 R v$ decreased by antiserum from the rabbits immunized with BCG, Salmonella rutgers, or crystalline egg albumin..$^{5) 6}$ Immune monocytes obtained from the rabbit vaccinated with $B C G$ resisted the degeneration caused by infection with $H 37 R v$ when maintained in immune serum (anti-BCG, anti-Salmonella, anti-ovalbumin).

But immune monocytes derived from the rabbit vaccinated with BCG were degenerated by $H 37 R v$ infection when cultivated in normal rabbit serum. Immune monocytes derived from animals immunized with S. rutgers showed early and marked degeneration by $H 37 R v$ infection even when cultivated in anti-BCG or anti-Salmonella serum..$^{\text {s)-8) }}$

In earlier reports ${ }^{2,4), 8}$ by the present authors it was described that mononuclear phagocytes obtained from the abdominal cavity, liver or subcutaneous tissue of mouse super-immunized with live vaccine of $S$. enteritidis inhibited intracellular multiplication of a 
virulent strain 116-54 of the same organism regardless of the presence of antibody in cell culture medium. In a previous paper, monocytes super-immunized with live vaccine of $S$. enteritidis inhibited intracellular growth of S.typhimurium, S. choleraesuis, and E. coli 0-55, regardless of the presence of immune sera, whereas the cells were degenerated by infection with $M$. tuberculosis $H 37 R v$. In the course of the study of infection of mouse with Salmonellae group the following results were obtained.

Summary. Mouse super-immunized with live vaccine of S. choleraesuis or $S$. typhimurium survived the intravenous infection with $10 \mathrm{MLD}$ virulent strain of $S$. enteritidis. In the course of the study of in vitro infection with $S$. enteritidis, it was found that mononuclear phagocytes derived from the abdominal cavity of mouse immunized with live vaccine of $S$. choleraesuis or S. typhimurium inhibited intracellular multiplication of bacteria and resisted the degeneration caused by infection, regardless of the presence of immune sera.

\section{References}

1) Sato, I., T. Tanaka, K. Saito, and S. Mitsuhashi: Proc. Japan Acad., 38, 133-138 (1962).

2) Mitsuhashi, S., M. Kawakami, H. Hashimoto, I. Sato, and T. Tanaka: Proc. Japan Acad., 37, 163-168 (1961).

3) Mitsuhashi, S., I. Sato, and T. Tanaka: J. Bacteriol., 81, 863-868 (1961).

4) Sato, I., T. Tanaka, K. Saito, and S. Mitsuhashi: Proc. Japan Acad., 37, 261-266 (1961).

5) Fong, J., P. Schneider, and S. S. Elberg: J. Exp. Med., 105, 25-37 (1957).

6) Elberg, S. S., P. Schneider, and J. Fong: J. Exp. Med., 106, 545-554 (1957).

7) Elberg, S. S.: Bacteriol. Rev., 24, 67-95 (1960).

8) Sato, I., T. Takaka, K. Saito, and S. Mitsuhashi: J. Bacteriol. (1962), in press. 